Technology Development

Division

Technology Development

Division

Technology Develowment

Division

Technology Development Division

Technology Development Division

Technology Development RECEIVEO JUL $0>2000$
$O S T I$

\author{
Fully Developed \\ Magnetohydrodynamic Flow \\ in a Rivulet
}

Technolloyy Dewelopment.

Division

Technology Development

ohvision

Technology Development:

Division

Techmology Development

Diviliston

Technology Development

Divislon

Technology Dovelopment

blvision

Tochmology Dovelopment

Division

Technology Development

Division

Technology Development

Division

Techmology Development

Division

Techmology Development

Drviston

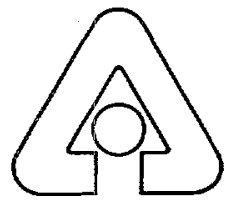

Argonne National Laboratory, Argonne, Illinois 60439

operated by The University of Chicago

for the United States Department of Energy under Contract W-31-109-Eng-38

Fusion Power program

Fusion Power Program

Fusion Power Proguram

Fusion Power Program

Fuslon pover Program

Fuslon Power Program

Fusion Power Program

Fuslon Power Program 
Argonne National Laboratory, with facilities in the states of Illinois and Idaho, is owned by the United States government, and operated by The University of Chicago under the provisions of a contract with the Department of Energy.

\section{DISCLAIMER}

This report was prepared as an account of work sponsored by an agency of the United States Government. Neither the United States Government nor any agency thereof, nor The University of Chicago, nor any of their employees or officers, makes any warranty, express or implied, or assumes any legal liability or responsibility for the accuracy, completeness, or usefulness of any information, apparatus, product, or process disclosed, or represents that its use would not infringe privately owned rights. Reference herein to any specific commercial product, process, or service by trade name, trademark, manufacturer, or otherwise, does not necessarily constitute or imply its endorsement, recommendation, or favoring by the United States Government or any agency thereof. The views and opinions of document authors expressed herein do not necessarily state or reflect those of the United States Government or any agency thereof, Argonne National Laboratory, or The University of Chicago.

Available electronically at http://www.doe.gov/bridge

Available for a processing fee to U.S. Department of Energy and its contractors, in paper, from:

U.S. Department of Energy

Office of Scientific and Technical Information

P.O. Box 62

Oak Ridge, TN 37831-0062

phone: (865) 576-8401

fax: (865) 576-5728

email: reports@adonis.osti.gov 


\section{DISCLAIMER}

Portions of this document may be illegible in electronic image products. Images are produced from the best available original document. 


\title{
FULLY DEVELOPED MAGNETOHYDRODYNAMIC FLOW IN A RIVULET
}

\author{
by \\ S. Molokov, Coventry University \\ C. B. Reed, Argonne National Laboratory
}
Argonne National Laboratory
9700 South Cass Avenue

Argonne, IL 60439

Work supported by the

Office of Fusion Energy Sciences

U.S. Department of Energy

Under Contract W-31-109-ENG-38 


\section{Contents}

Abstract 2

1. Introduction.................................. 3

2. Formulation................................. 4

2.1 Problem I...................................... 5

2.2 Problem II..................................... 6

2.3 Solution procedure............................... 8

3. Magnetic field transverse to the plate............ 9.

3.1 The core C..................................... 9

3.2 Hartmann layer at the free surface HF............ 11

3.3 Composite expansion............................. 13

3.4 Results....................................... 13

4. Magnetic field parallel to the plate.............. 15

4.1 The core C...................................... 15

4.2 Hartmann layer at the free surface HS............ 17

4.3 Further discussion of the core.................... 18

4.4 Results....................................... 19

5. Conclusions................................ 21

6. References.................................. 22

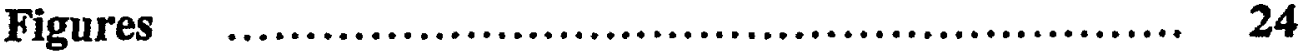




\title{
FULLY DEVELOPED MAGNETOHYDRODYNAMIC FLOW IN A RIVULET
}

\section{S. Molokov and C.B. Reed}

\begin{abstract}
Fully developed magnetohydrodynamic flow in a rivulet on an inclined solid plate is considered. The plate is partially wetted, and is electrically insulating. The external magnetic field is transverse to the flow direction, and is either transverse or parallel to the plate. An asymptotic solution of the problem for high values of the Hartmann number is obtained. If the magnetic field is transverse to the plate, the flow in the core is determined by the balance of the Lorentz force and the gravity. The Hartmann layer is formed at the plate, which allows high electric current to circulate in the rivulet's cross-section. This leads to the reduction of the fluid velocity; the latter being proportional to $B_{0}^{-1}$, where $B_{0}$ is the induction of the external field. If the magnetic field is parallel to the plate, magnetic field lines cross the free surface only. The Lorentz force alone cannot balance the gravity, and the core is viscous. The fluid velocity is independent of the magnetic field induction, and is of the same order as that in a flow without the field. For both field directions (i) the velocity does not vary along the magnetic field lines; (ii) there is a lower-order Hartmann layer at the free surface, which is formed to relax traction at the free surface, produced by the core flow.
\end{abstract}




\section{INTRODUCTION}

In recent years there has been an increased interest to free surface magnetohydrodynamic (MHD) flows in the context of liquid metal divertors for tokamak fusion reactors [1]. An essential element of such a divertor is a system of liquid-metal films, jets, or drops. The role of this system is to absorb a very high flux of energy and charged particles from the plasma region of the reactor. The divertor operates in a very strong magnetic field, and this results in several MHD-related problems. A review of the relevant experimental and theoretical work, together with the classification of the most important MHD problems has been given recently by Molokov \& Reed [2].

In a divertor concept employing a liquid-metal film, the latter appears from the orifice and flows down an inclined solid plate. The film may or may not be supported laterally by the sidewalls. It is a well-known experimental fact that wettability of the plate plays a very important role in determining the nature of the flow [3]-[6]. If wettability of the sidewalls and/or the plate is poor, and if in addition the film is sufficiently thin, or the flow rate is low, the film detaches from the sidewalls and forms one or several rivulets after leaving the orifice. Rivulets also form if one or several jets impact on an inclined plate [7].

If the plate is long enough, the flow downstream tends to become fully developed and driven by gravity only. In this initial investigation we employ matched asymptotic expansions for high values of the Hartmann number to study this fully developed flow regime. The magnetic field is supposed to be transverse to the flow and either transverse or parallel to the plate. The latter case is more relevant to the divertor applications.

If the fluid layer is infinite in the direction of the parallel magnetic field, the gradient of the electric potential fully balances the induced electromotive force, and no electric current is induced. For a rivulet, however, lines of force of the parallel field intersect the free surface. It is shown that even though they do not intersect a solid wall, an electric current is induced. This plays an important role in the flow redistribution over a rivulet's cross-section. The fluid velocity, however, is practically independent of the induction of the applied field. 


\section{FORMULATION}

Consider the steady, gravity-driven, isothermal, fully developed flow of a viscous, electrically conducting, incompressible fluid along the $x_{*}$-axis in a rivulet (figure 1). A solid plate is located at $z_{*}=0$; it is inclined at an angle $\alpha$ to the horizon. Here $\left(x_{*}, y_{*}, z_{*}\right)$ are Cartesian co-ordinates. Dimensional quantities are denoted by letters with asterisks, while their dimensionless counterparts - with the same letters, but without the asterisks. A strong, uniform, transverse magnetic field $\mathbf{B}_{0 *}=B_{0} \mathbf{e}_{B}$ is applied in the plane $\left(y_{*}, z_{*}\right)$ at an angle $\gamma$ to the horizon in the direction of a unit vector $e_{B}$. The location of the free surface is defined either by the equation

$$
z_{*}=h_{*}\left(y_{*}\right)
$$

(Sec. 3, transverse field), or equivalently, by

$$
y_{*}= \pm f_{*}\left(z_{*}\right) \text { for } y_{*}>0 \text { and } y_{*}<0 \text {, respectively }
$$

(Sec. 4, parallel field). This is a matter of convenience only. Neither function $h_{*}\left(y_{*}\right)$, nor $f_{*}\left(z_{*}\right)$ is known in advance.

Since all the flow quantities are independent of $x_{*}$, the magnetohydrodynamic equations governing the problem are [8]:

$$
\begin{gathered}
\rho v \nabla_{*}^{2} u_{*}+B_{0}\left[j_{y *} \sin \gamma-j_{z *} \cos \gamma\right]=-\rho g \sin \alpha \\
-j_{y_{*}} b_{*}=\frac{\partial p_{*}}{\partial z_{*}}+\rho g \cos \alpha \\
j_{z *} b_{*}=\frac{\partial p_{*}}{\partial y_{*}}, \\
j_{y^{*}}=\mu^{-1} \frac{\partial b_{*}}{\partial z_{*}}, \\
j_{z *}=-\mu^{-1} \frac{\partial b_{*}}{\partial y_{*}}, \\
j_{y_{*}}=\sigma\left[-\frac{\partial \phi_{*}}{\partial y_{*}}-u_{*} B_{0} \sin \gamma\right] \\
\left.j_{z *}=\sigma-\frac{\partial \phi_{*}}{\partial z_{*}}+u_{*} B_{0} \cos \gamma\right] \\
\frac{\partial j_{y_{*}}}{\partial y_{*}}+\frac{\partial j_{z *}}{\partial z_{*}}=0,
\end{gathered}
$$


where $u_{*}$ and $b_{*}$ are the $x_{*}$-components of the fluid velocity and the induced magnetic field, respectively, $p_{0}$ is the pressure, $\mathbf{j}_{.}=j_{y}, \hat{\mathbf{y}}+j_{z} \hat{\mathbf{z}}$ is the electric current density, $\phi_{*}$ is the electric potential; they are functions of $y_{*}$ and $z_{*}$ only; $\nabla_{*}=\hat{\mathbf{y}} \partial / \hat{y}_{*}+\hat{\mathbf{z}} \partial / z_{*}$.

The characteristic values of the length, the fluid velocity, the induced magnetic field, the electric current density, the electric potential, and the pressure are $a_{*}$, the maximum height, $v_{0}=\rho g \sin \alpha /\left(\sigma B_{0}^{2}\right), \sigma \mu a_{*} v_{0} B_{0}, \sigma v_{0} B_{0}, a_{*} v_{0} B_{0}$, and $a_{4} \rho g \cos \alpha$, respectively. In the above, $\sigma, \rho, v$ are the electrical conductivity, density and kinematic viscosity of the fluid; $\mu$ is the magnetic permeability; $g$ is the intensity of gravity. There are several possible choices for the characteristic length, $a_{*}$. Here we assume that it is the height of the rivulet, which is experimentally measured.

From the equations above follows that they can be arranged into two groups. The distribution of the fluid velocity, current, and electric potential (designated as Problem I) is governed by Eqs. (2.1), (2.6)-(2.8), while that of the pressure, the induced magnetic field, and the location of the free surface (Problem II) is determined by Eqs. (2.2)-(2.5). They are considered in Secs. 2.1 and 2.2, respectively.

\subsection{Problem I}

After normalization, Eqs. (2.1), (2.6)-(2.8) become

$$
\begin{gathered}
H a^{-2} \nabla^{2} u+j_{y} \sin \gamma-j_{z} \cos \gamma=-1, \\
j_{y}=-\frac{\partial \phi}{\partial y}-u \sin \gamma, \\
j_{z}=-\frac{\partial \phi}{\partial z}+u \cos \gamma, \\
\frac{\partial_{y}}{\partial y}+\frac{\partial_{z}}{\partial z}=0,
\end{gathered}
$$

where $H a=a_{*} B_{0} \sqrt{\sigma / \rho v}$ is the Hartmann number, which is supposed to be sufficiently high.

Consider now the boundary conditions at the solid wall. For the fluid velocity the noslip condition holds. Although it is possible to construct an asymptotic solution of the problem for a plate having arbitrary electrical conductivity and thickness, for the sake of clarity in this initial investigation we restrict ourselves to the case of an electrically insulating plate. Then the boundary conditions for the fluid velocity and current at the solid wall are

$$
u=0, j_{z}=0 \quad \text { at } z=0 .
$$

At the free surface both the tangential stress and the normal component of current vanish. This gives 


$$
\frac{\partial u}{\partial n}=0, \quad \mathbf{j} \cdot \hat{\mathbf{n}}=0 \quad \text { at the free surface }
$$

where $\hat{\mathbf{n}}$ is the outward normal unit vector to the free surface.

\subsection{Problem II}

Substituting Eqs. (2.4), (2.5) into (2.2), (2.3), integrating the latter equations, and introducing the dimensionless variables gives the expression for pressure as follows:

$$
p=-z+p_{0}-G b^{2} \text {. }
$$

The first two terms in the right-hand side of Eq. (2.15) represent the hydrostatic pressure; $p_{0}=$ const. being its as yet unknown value at $z=0$. The third term represents the magnetic pressure induced by the fluid motion, where

$$
G=\frac{\mu a_{*} \rho g \sin ^{2} \alpha}{B_{0}^{2} \cos \alpha}
$$

is the dimensionless parameter, which measures the ratio of the gravitational to magnetic pressure.

Suppose that the location of the free surface is given by the equation $z=h(y)$. At the free surface the jump in pressure is balanced by the surface tension [9], [10]. This gives:

$$
\left(p-p_{a}\right) B_{o} \operatorname{cs} \alpha=-\frac{d^{2} h}{d y^{2}}\left[1+\left(\frac{d h}{d y}\right)^{2}\right]^{-3 / 2} \text { at } z=h(y)
$$

where $p_{a}=$ const. is the dimensionless pressure of the surrounding medium, which has been assumed to be dynamically passive, and

$$
B_{o}=a_{*}^{2} \rho g / T
$$

is the Bond number, which characterises the ratio of gravitational to surface tension forces. In the above, $T$ is the surface tension coefficient.

As follows from Eq. (2.15), the magnetic pressure leads to the deviation of the hydrodynamic from the hydrostatic pressure, which is linear in $z$. However, in a fully developed flow the induced magnetic field vanishes at the electrically insulating boundaries [8]. Hence the magnetic pressure vanishes at both the solid plate and the free surface. The latter fact means that the location of the free surface is exactly the same as that in a nonmagnetic case [9], [10] for an arbitrary magnetic Reynolds number provided the flow is stable. Indeed, substituting (2.15) into (2.16) gives

$$
\frac{d^{2} h}{d y^{2}}\left[1+\left(\frac{d h}{d y}\right)^{2}\right]^{-3 / 2}=k h-\delta
$$


where

$$
\begin{gathered}
k=B_{a} \cos \alpha, \\
\delta=\left(p_{0}-p_{a}\right) B_{a} \cos \alpha .
\end{gathered}
$$

Eq. (2.17) is solved subject to the boundary and symmetry conditions

$$
\begin{gathered}
h=1, \frac{d h}{d y}=0 \text { at } y=0, \\
h=0, \frac{d h}{d y}=-\tan \theta \text { at } y=d .
\end{gathered}
$$

Here $\theta$ is the static contact angle assumed to be sharp $\left(0<\theta \leq \frac{1}{2} \pi\right)$.

From (2.17)-(2.21) follows:

$$
\delta=\frac{1}{2} k+1-\cos \theta,
$$

and therefore, the pressure at the solid plate is

$$
p_{0}=p_{a}+\frac{1}{2}+\frac{1-\cos \theta}{B_{o} \cos \alpha} \text {. }
$$

The solution to the problem defined by Eqs. (2.17)-(2.21) can be expressed in parametric form as follows [9]:

$$
y=\tilde{y}(\omega), h=\widetilde{z}(\omega),
$$

where

$$
\begin{gathered}
\widetilde{z}(\omega)=\frac{\delta}{k}-\frac{2 \sqrt{k}}{s}\left[1-s^{2} \sin ^{2} \omega\right]^{1 / 2}, \\
\tilde{y}(\omega)=\frac{2 \sqrt{k}}{s}\left\{E(\omega, s)-E\left(\frac{1}{2} \pi, s\right)-\left(1-\frac{1}{2} \omega^{2}\right)\left[F(\omega, s)-F\left(\frac{1}{2} \pi, s\right)\right]\right\},
\end{gathered}
$$

where $\frac{1}{2} \pi-\frac{1}{2} \theta \leq \omega \leq \frac{1}{2} \pi$ is a parameter $\left(\omega=\frac{1}{2} \pi-\frac{1}{2} \theta\right.$ at $y=d ; \omega=\frac{1}{2} \pi$ at $\left.y=0\right), E(, \cdot)$ and $F($,$) are the elliptic integrals of the first and second kind, respectively, and$

$$
s=\frac{2 \sqrt{k}}{\sqrt{4 k+(\delta-k)^{2}}} .
$$

For calculations of the flow variables the following relations are used:

$$
\frac{d h}{d y}=\tan 2 \omega, \frac{d^{2} h}{d y^{2}}=\frac{\delta-k h}{\cos ^{3} 2 \omega} .
$$

The graphs of $h(y)$ for $k=0.01$, and for three different values of the contact angle are shown in fig. 2. 


\subsection{Solution procedure}

The solution to the problem of the flow in a rivulet is constructed in the following way. First, the location of the free surface is determined from expressions (2.24)-(2.26). Next, Problem I is solved to determine the fluid velocity, electric current density and electric potential. Then the induced magnetic field is determined from either of the dimensionless versions of Eqs. (2.4) and (2.5), namely

$$
\frac{\partial b}{\partial y}=-j_{z}, \quad \frac{\partial b}{\partial z}=j_{y} .
$$

Finally, pressure is obtained from Eq. (2.15), if necessary.

Several useful integral relations can be obtained from Eqs. (2.9)-(2.14). In particular, integrating Eq. (2.9) over the rivulet's cross-section, and applying the divergence theorem gives the relation to be used in Sec. 4:

$$
\left.\int_{-d}^{d} \frac{a d}{\partial z}\right|_{z=0} d y=H a^{2} A,
$$

where

$$
A=2 \int_{0}^{1} h(y) d y
$$

is the cross-sectional area. This means that in dimensional terms the total traction exerted by the plate on the fluid is independent of the induction of the magnetic field, and is the same as that in a non-magnetic case. This is owing to the well-known fact that for electrically insulating boundaries currents complete their loop in the fluid, so that the total current in the fluid is zero. Globally, the Lorentz force does not affect the friction. It leads only to the redistribution of the fluid velocity in the rivulet's cross-section in that it makes it constant in the direction of the field (see below).

In Secs. 3 and 4 Problem I is solved using matched asymptotic expansions at high values of the Hartmann number for the magnetic field transverse and parallel to the plate, respectively. In both of these cases the problem is symmetric with respect to $y$. It will be considered for $y>0$ only subject to specified symmetry conditions at $y=0$. The expressions for $b$ are also derived to plot the electric current lines, since $b$ is the stream function for the electric current. 


\section{MAGNETIC FIELD TRANSVERSE TO THE PLATE}

Consider first the flow in a field transverse to the plate, i.e. for $\gamma=\frac{1}{2} \pi ; \mathbf{e}_{B}=\hat{\mathbf{z}}$. The free surface is defined by the equation $z=h(y)$. Eqs. (2.7)-(2.10) become

$$
\begin{gathered}
H a^{-2} \nabla^{2} u+j_{y}=-1, \\
j_{y}=-\frac{\partial \phi}{\partial y}-u, \\
j_{z}=-\frac{\partial \phi}{\partial z}, \\
\frac{\partial_{y}}{\partial y}+\frac{\partial_{z}}{\partial z}=0 .
\end{gathered}
$$

These equations are solved subject to the boundary conditions (2.13), (2.14), where the normal unit vector is defined as

$$
\hat{\mathbf{n}}=l^{-1}\left[-h^{\prime} \hat{\mathbf{y}}+\hat{\mathbf{z}}\right] ; \quad l(y)=\sqrt{1+h^{\prime 2}} .
$$

Here and elsewhere in Sec. $3,^{\prime}=d / d y$.

The symmetry conditions are

$$
\frac{\partial u}{\partial y}=0, \phi=0 \text { at } y=0
$$

In a strong magnetic field four main flow subregions are expected (figure 3):

- the core $C$

- the Hartmann layer $H P$ of thickness $O\left(H a^{-1}\right)$ at the plate;

- the Hartmann layer $H F$ of thickness $O\left(H a^{-1}\right)$ at the free surface;

- the edge layers $T$; their dimensions depend on the value of the contact angle. For a sharp contact angle, $\theta$, these layers are not important.

\subsection{The core $C$}

In the core there is a balance between the Lorentz force and the gravity, while viscous terms in Eq. (3.1) may be neglected. Then Eq. (3.1) indicates that the current in the core is $O(1)$. The core is surrounded by two Hartmann layers, $H P$ and $H F$. One of them is at the plate, while the other is at the free surface. Therefore, the core current must pass through one or the other Hartmann layer, or both. Conservation of current requires that the current is $O(H a)$ in a layer, and by passing through the layer, the current induces an electric potential difference $O(H a)$. This electric potential in turn induces the $O(H a)$-core velocity. This mechanism is characteristic of flows with electrically insulating boundaries; it is well known 
in duct flows [8] and buoyant convection in a horizontal magnetic field transverse to the temperature gradient [11]-[13]. Therefore, in the core we are looking for the asymptotic expansions in the form

$$
\begin{aligned}
& u=H a u_{C}^{(-1)}+u_{C}^{(0)}+H a^{-1} u_{C}^{(1)}+\ldots, \\
& \phi=H a \phi_{C}^{(-1)}+\phi_{C}^{(0)}+H a^{-1} \phi_{C}^{(1)}+\ldots, \\
& \mathbf{j}=\mathrm{j}_{C}^{(0)}+H a^{-1} \mathbf{j}_{C}^{(1)}+H a^{-2} \mathbf{j}_{C}^{(2)}+\ldots .
\end{aligned}
$$

Throughout this paper subscripts $C, H P, H F, H S, P$ and $T$ of the flow variables denote the flow subregion, where corresponding limit equations are valid. Variables with these subscripts are $O(1)$.

We are interested in the asymptotic solution valid to $O(1)$ in both the core and the Hartmann layers. To this order the general solution in the core is

\section{Order $H a^{1}$}

$$
\phi_{C}^{(-1)}=\phi_{C}^{(-1)}(y), \quad u_{c}^{(-1)}=-\frac{d \phi_{C}^{(-1)}}{d y},
$$

$\underline{\text { Order } H a^{0}}$

$$
\begin{gathered}
j_{y, C}^{(0)}=-1, \quad j_{z, C}^{(0)}=j_{z, C}^{(0)}(y), \\
\phi_{C}^{(0)}=-z j_{z, C}^{(0)}(y)+\phi_{0}(y), \quad u_{C}^{(0)}=z \frac{d j_{z, C}^{(0)}}{d y}-\frac{d \phi_{0}}{d y}+1,
\end{gathered}
$$

$\underline{\text { Order } \mathrm{Ha}^{-1}}$

$$
j_{y, C}^{(1)}=\frac{d^{3} \phi_{C}^{(-1)}}{d y^{3}}, j_{z, C}^{(1)}=-z \frac{d^{4} \phi_{C}^{(-1)}}{d y^{4}}+j_{0}(y)
$$

where unknown functions $\phi_{C}^{(-1)}, \phi_{0}, j_{2, C}^{(0)}, j_{0}$ are to be determined from matching conditions with the Hartmann layers.

The Hartmann layer $H P$ at the solid plate has a local exponential structure. Its analysis is standard [14], and will not be discussed in detail here. Its contribution to the composite asymptotic expansions is taken into account in Sec. 3.3. The solution in the layer provides two matching conditions for the core variables:

$$
j_{z, C}^{(0)}=\frac{d^{2} \phi_{C}^{(-1)}}{d y^{2}}, \quad j_{z, C}^{(1)}=\frac{d^{2} \phi_{C}^{(0)}}{d y^{2}} \text { at } z=0 .
$$

The other two matching conditions are provided by the Hartmann layer $H F$, which requires a more detailed analysis. 


\subsection{Hartmann layer at the free surface $H F$}

The Hartmann-layer variables are

$$
z_{H F}=H a[z-h(y)], \quad y_{H F}=y .
$$

The governing equations (3.1)-(3.4) are transformed into

$$
\begin{gathered}
l^{2}\left(y_{H F}\right) \frac{\partial^{2} u}{\partial z_{H F}^{2}}-H a^{-1}\left[h^{\prime \prime}\left(y_{H F}\right) \frac{\partial u}{\partial z_{H F}}+2 H\left(y_{H F}\right) \frac{\partial^{2} u}{\partial y_{H F} \partial_{H F}}\right]+H a^{-2} \frac{\partial^{2} u}{\partial z_{H F}^{2}}+j_{y}=-1 \\
j_{y}=H a h^{\prime}\left(y_{H F}\right) \frac{\partial \phi}{\partial z_{H F}}-\frac{\partial \phi}{\partial y_{H F}}-u \\
j_{z}=-H a \frac{\partial \phi}{\partial z_{H F}} \\
H a\left[\frac{\partial_{z}}{\partial z_{H F}}-h\left(y_{H F}\right) \frac{\partial_{y}}{\partial z_{H F}}\right]+\frac{\partial y_{y}}{\partial y_{H F}}=0
\end{gathered}
$$

The boundary conditions (2.14) at the free surface are

$$
H a l^{2}\left(y_{H F}\right) \frac{\partial u}{\partial z_{H F}}-h^{\prime}\left(y_{H F}\right) \frac{\partial u}{\partial y_{H F}}=0, j_{z}-h^{\prime}\left(y_{H F}\right) j_{y}=0 \quad \text { at } z_{H F}=0
$$

As has been discussed above, the electric currents, and thus all the flow variables in the Hartmann layer are $O(1)$. Therefore, in layer $H F$ we are looking for the asymptotic expansions in the form

$$
\begin{aligned}
& u=H a u_{H F}^{(-1)}+u_{H F}^{(0)}+H a^{-1} u_{H F}^{(1)}+\ldots, \\
& \phi=H a \phi_{H F}^{(-1)}+\phi_{H F}^{(0)}+H a^{-1} \phi_{H F}^{(1)}+\ldots, \\
& \mathbf{j}=H a \mathbf{j}_{H F}^{(-1)}+\mathrm{j}_{H F}^{(0)}+H a^{-1} \mathbf{j}_{H F}^{(1)}+\ldots .
\end{aligned}
$$

Substituting these expressions into Eqs. (3.12)-(3.16) gives the following solutions:

$\underline{\text { Order } H a^{1}}$

$$
\begin{gathered}
\phi_{H F}^{(-1)}=\phi_{C}^{(-1)}\left(y_{H F}\right), \\
u_{H F}^{(-1)}=u_{C}^{(-1)}\left(y_{H F}\right), \\
j_{y, H F}^{(-1)}=0, \\
j_{z, H F}^{(-1)}=0,
\end{gathered}
$$




\section{Order $H a^{0}$}

$$
\begin{gathered}
\phi_{H F}^{(0)}=\left.\phi_{C}^{(0)}\right|_{z=h(y)}=-h j_{z, C}^{(0)}+\phi_{0}, \\
u_{H F}^{(0)}=l^{2}\left(y_{H F}\right)-\left.\frac{\partial \phi_{C}^{(0)}}{\partial y_{H F}}\right|_{z=h(y)}-h^{\prime}\left(y_{H F}\right) \frac{\partial^{2} \phi_{C}^{(-1)}}{\partial y_{H F}^{2}} \exp \left[\frac{z_{H F}}{l^{2}\left(y_{H F}\right)}\right], \\
j_{y, H F}^{(0)}=-1+\frac{h^{\prime}\left(y_{H F}\right)}{l^{2}\left(y_{H F}\right)} \cdot \frac{\partial^{2} \phi_{C}^{(0)}}{\partial y_{H F}^{2}} \exp \left[\frac{z_{H F}}{l^{2}\left(y_{H F}\right)}\right], \\
j_{z, H F}^{(0)}=-h^{\prime}\left(y_{H F}\right)+\frac{h^{2}\left(y_{H F}\right)}{l^{2}\left(y_{H F}\right)} \cdot \frac{\partial^{2} \phi_{C}^{(0)}}{\partial_{H F}^{2}} \exp \left[\frac{z_{H F}}{l^{2}\left(y_{H F}\right)}\right],
\end{gathered}
$$

Matching with the core variables gives

$$
j_{z, C}^{(0)}(y)=-h^{\prime}(y)
$$

which determines the $z$-component of the core current directly. Finally, the fourth matching condition is obtained from the analysis of the $O\left(\mathrm{Ha}^{-1}\right)$-solution in layer $H F$. For this purpose it is sufficient to integrate over the layer the equation of the conservation of current

$$
\frac{\partial}{\partial z_{H F}}\left[j_{z, C}^{(1)}-h^{\prime}\left(y_{H F}\right) j_{y, C}^{(1)}\right]=-\frac{\partial_{y, C}^{(0)}}{\partial y_{H F}},
$$

which follows from Eq. (3.15). This gives

$$
j_{z, C}^{(1)}-h^{\prime}(y) j_{y, C}^{(1)}=\frac{\partial}{\partial y}\left[h(y) \frac{\partial^{2} \phi_{C}^{(-1)}}{\partial y^{2}}\right] \text { at } z=h(y) \text {. }
$$

Now, Eqs. (3.10) and (3.26) give the remaining three unknown functions in the form

$$
\begin{gathered}
\phi_{C}^{(-1)}(y)=-\int_{0}^{y} h(t) d t, \\
j_{0}(y)=-\left(h h^{\prime}\right)^{\prime \prime}, \\
\phi_{0}(y)=y-h h^{\prime} .
\end{gathered}
$$

While deriving expressions (3.28)-(3.30) the fact that the $y$-component of the total current is zero to order $H a^{-1}$ for an arbitrary $y$, i.e.

$$
I_{y}=\int_{0}^{h(y)} j_{y} d z=0
$$

has been used. This is necessary to determine the integration constants. 


\subsection{Composite expansions}

Now the solution in the core and both the Hartmann layers is known to $O(1)$. The composite asymptotic expansions of the flow variables (denoted by the superscript " $c$ "), which are valid in both the core and the two Hartmann layers are:

$$
\begin{gathered}
\phi^{c}=-H a \int_{0}^{y} h(t) d t+y+(z-h) h+\ldots, \\
u^{c}=\left\{H a h+\left(h h^{\prime}\right)^{\prime}\right\}[1-\exp (-H a z)]-z h^{\prime}+h^{2} \exp \left(H a \frac{z-h}{1+h^{2}}\right)+\ldots, \\
j_{y}^{c}=\left[H a h+\left(h h^{\prime}\right)^{\prime}\right] \exp (-H a z)-1-\frac{h^{2}}{1+h^{2}} \exp \left(H a \frac{z-h}{1+h^{\prime 2}}\right)-H a^{-1} h^{\prime \prime}+\ldots, \\
j_{z}^{c}=-h^{\prime}[1-\exp (-H a z)]-\frac{h^{3}}{1+h^{\prime 2}} \exp \left(H a \frac{z-h}{1+h^{2}}\right)+H a^{-1}\left[z h^{\prime \prime}-\left(h h^{\prime}\right)^{\prime \prime}\right]+\ldots .
\end{gathered}
$$

From Eqs. (2.28) and (3.34) follows the equation for the composite expansion for the induced magnetic field valid to $O\left(H a^{-1}\right)$ :

$$
\begin{aligned}
b^{c}= & \left\{h+\left(h h^{\prime}\right)^{\prime} H a^{-1}\right\}[1-\exp (-H a z)] \\
& -z\left(1+h^{\prime} H a^{-1}\right)-H a^{-1} h^{2} \exp \left(H a \frac{z-h}{1+h^{2}}\right)+\ldots .
\end{aligned}
$$

\subsection{Results}

When the fluid flows down an inclined plane in a transverse magnetic field, the $O(1)$ electric current is induced initially in the negative $y$-direction. This component of current interacts with the magnetic field to produce the Lorentz force, which balances gravity in the core. Since the traction at the free surface is zero, there is no Hartmann layer at the free surface to this order approximation. Hence, the core electric current must vanish at the free surface, and as a result, the current lines repeat the shape of the free surface (figure 4). The electric current completes its path in the Hartmann layer at the plate, where it is $O(H a)$ owing to current conservation. While passing through the Hartmann layer, the current induces an $O(H a)$ electric potential difference in both the layer, and the core, which must be balanced by the electromotive force $(u \hat{\mathbf{x}}) \times \mathbf{e}_{B}$. This leads to the $O(H a)$ velocity in the core. Figure 5 shows velocity profiles at different positions $y=$ const. To the leading order, $O(H a)$, the profile repeats the shape of the rivulet's cross-section, similar to the flow in insulating ducts [8]. The $O(1)$-correction to the velocity profile leads to a slight linear variation with $z$ in the core.

As has been discussed above, there is no Hartmann layer at the free surface to the leading order. The Hartmann layer appears in the next, $O(1)$ approximation. The reason for its appearance is that the $O(H a)$-velocity is a function of $y$ only, and this leads to a non-zero, 
$O$ (1) traction at the free surface. This traction is compensated by the exponential variation of the velocity in the Hartmann layer $H F$, which is evident in figure 5.

It is interesting to note that the Hartmann layer $H F$ vanishes at the symmetry plane $y=0$. The reason for this is evident: at $y=0$ the normal derivative, $\partial / \partial 2$, coincides with $\partial / \partial$, which is zero when applied to $u_{c}^{(-1)}$. This situation must be contrasted with the corresponding duct flow problem, where the strongest velocity gradient occurs exactly where the normal to the surface component of the magnetic field is strongest.

To the leading order the flow rate, $Q$, and the average velocity, $u_{a v}$, are

$$
\begin{gathered}
Q=2 H a \int_{0}^{d} h^{2}(y) d y, \\
u_{a v}=Q / A .
\end{gathered}
$$

Eqs. (3.33), (3.37) and (3.38) indicate that in dimensional terms both the discharge, and the local and average fluid velocities are proportional to $B_{0}^{-1}$. 


\section{MAGNETIC FIELD PARALLEL TO THE PLATE}

Suppose now that the magnetic field is parallel to the plate, i.e. $\gamma=0 ; \mathbf{e}_{B}=\hat{\mathbf{y}}$. It is convenient to define the location of the free surface in a different way to that in Sec. 3, namely by the equations $y= \pm f(z)$ for $y>0$ and $y<0$, respectively (in a parametric form defined in Sec. 2.1, $f= \pm \widetilde{y}(\omega), z=\widetilde{z}(\omega)$ ). Then Eqs. (2.9)-(2.12) become

$$
\begin{gathered}
H a^{-2} \nabla^{2} u-j_{z}=-1, \\
j_{y}=-\frac{\partial \phi}{\partial y}, \\
j_{z}=-\frac{\partial \phi}{\partial z}+u, \\
\frac{\partial j_{y}}{\partial y}+\frac{\partial_{z}}{\partial z}=0 .
\end{gathered}
$$

The boundary conditions for the system of Eqs. (4.1)-(4.4) are given by (2.13), (2.14), where the normal unit vector is defined as

$$
\hat{\mathbf{n}}=L^{-1}\left[\hat{\mathbf{y}}-f^{\prime} \hat{\mathbf{z}}\right] ; L(z)=\sqrt{1+f^{\prime 2}}
$$

Here and elsewhere in Sec. $4,^{\prime}=d / d z$.

The symmetry conditions are

$$
\frac{\partial u}{\partial y}=0, \quad j_{y}=0 \quad \text { at } y=0
$$

In a strong magnetic field three main types of flow subregions are expected (figure 6):

- the core $C$

- the Hartmann layer $H S$ of thickness $O\left(H^{-1}\right)$ at the free surface;

- the parallel layer $P$ of thickness $O\left(H a^{-1 / 2}\right)$ at the plate;

The Hartmann layer at the free surface is denoted as $H S$, since the boundary-layer variables are different from those in layer $H F$ (Sec. 3). We restrict ourselves to the determination of the leading terms in the asymptotic expansions in each flow subregion.

\subsection{The core $C$}

Let us discuss the scaling first. If the viscous term in the Eq. (4.1) is neglected, then $j_{z}=1$. Eq. (4.4), together with the second symmetry condition in (4.5), gives $j_{y}=0$. This means that there is a non-zero normal current into the Hartmann layer $H S$, which implies the $O(H a)$ current in the layer in the tangential direction to the free surface. This current interacts 
with the applied field to produce the Lorentz force in the layer, which must be balanced by the viscous term, and this implies velocity $O(\mathrm{Ha})$ in the layer. The Hartmann layer appears only if the traction at the free surface is non-zero. Therefore, according to the mechanism discussed in Sec. 3, the first term in the asymptotic expansion of the fluid velocity in the layer, and thus in the core, must be even higher, namely $O\left(\mathrm{Ha}^{2}\right)$. But this means (cf. Eq. (4.1)) that the viscous term in the core is of the same order as the other two. Therefore, for the parallel field, the balance between the Lorentz force and the gravity alone is inappropriate. Therefore, the appropriate expansions in the core are:

$$
\begin{aligned}
& u=H a^{2} u_{C}^{(-2)}+H a u_{C}^{(-1)}+u_{C}^{(0)}+\ldots, \\
& \phi=H a^{2} \phi_{C}^{(-2)}+H a \phi_{C}^{(-1)}+\phi_{C}^{(0)}+\ldots \\
& \mathbf{j}=\mathbf{j}_{C}^{(0)}+H a^{-1} \mathbf{j}_{C}^{(1)}+H a^{-2} \mathbf{j}_{C}^{(2)}+\ldots
\end{aligned}
$$

The core equations for the leading terms are

$$
\begin{gathered}
\nabla^{2} u_{c}^{(-2)}-j_{z, C}^{(0)}=-1, \\
0=\frac{\partial \phi_{C}^{(-2)}}{\partial y}, \\
u_{C}^{(-2)}=\frac{\partial \phi_{C}^{(-2)}}{\partial z}, \\
\frac{\partial_{y, C}^{(0)}}{\partial y}+\frac{\partial_{z, C}^{(0)}}{\partial z}=0 .
\end{gathered}
$$

Eqs. (4.6)-(4.9) indicate that to the leading order, the fluid velocity, the electric potential, and the $z$-component of current are functions of $z$ only. This yields

$$
\begin{gathered}
\frac{d^{2} u_{C}^{(-2)}}{d z^{2}}-j_{z, C}^{(0)}=-1, \\
u_{C}^{(-2)}=\frac{d \phi_{C}^{(-2)}}{d z}, \\
j_{y, C}^{(0)}=-y \frac{d j_{z, C}^{(0)}}{d z} .
\end{gathered}
$$

There are two unknown functions of $z$ in the core, namely $\phi_{C}^{(-2)}$ and $j_{z, C}^{(-2)}$, which are determined by matching with the Hartmann layer at the free surface. 


\subsection{Hartmann layer at the free surface $H S$}

The Hartmann layer $H S$ can be treated in the same way as layer $H F$ in Sec. 3. It is more convenient, however, to use different stretching as follows:

$$
y_{H S}=H a[y-f(z)], \quad z_{H S}=z .
$$

Then the governing equations (4.1)-(4.4) are transformed into

$$
\begin{gathered}
L^{2}\left(z_{H S}\right) \frac{\partial^{2} u}{\partial y_{H S}^{2}}-H a^{-1}\left[f^{\prime}\left(z_{H S}\right) \frac{\partial u}{\partial y_{H S}}+2 f^{\prime}\left(z_{H S}\right) \frac{\partial^{2} u}{\partial y_{H S} \partial z_{H S}}\right]+H a^{-2} \frac{\partial^{2} u}{\partial z_{H S}^{2}}-j_{z}=-1, \\
j_{y}=-H a \frac{\partial \phi}{\partial y_{H S}}, \\
j_{z}=H a f^{\prime}\left(z_{H S}\right) \frac{\partial \phi}{\partial y_{H S}}-\frac{\partial \phi}{\partial z_{H S}}+u, \\
H\left[\frac{\partial_{y}}{\partial y_{H S}}-f\left(z_{H S}\right) \frac{\partial_{z}}{\partial y_{H S}}\right]+\frac{\partial_{z}}{\partial z_{H S}}=0 .
\end{gathered}
$$

The boundary conditions at the free surface are

$$
H a L^{2}\left(z_{H S}\right) \frac{\partial u}{\partial y_{H S}}-f^{\prime}\left(z_{H S}\right) \frac{\partial u}{\partial z_{H S}}=0, j_{y}-f^{\prime}\left(z_{H S}\right) j_{z}=0 \quad \text { at } y_{H S}=0
$$

The orders of magnitude of the fluid velocity and the electric potential are the same as those in the core, while the current density is $O(H a)$ times higher. This leads to the expansions

$$
\begin{gathered}
u=H a^{2} u_{H S}^{(-2)}+H a u_{H S}^{(-1)}+u_{H S}^{(0)}+\ldots, \\
\phi=H a^{2} \phi_{H S}^{(-2)}+H a \phi_{H S}^{(-1)}+\phi_{H S}^{(0)}+\ldots, \\
\mathbf{j}=H a \mathbf{j}_{H S}^{(-1)}+\mathbf{j}_{H S}^{(0)}+H a^{-1} \mathbf{j}_{H S}^{(1)}+\ldots
\end{gathered}
$$

Substituting these expansions into Eqs. (4.14)-(4.18) gives:

Order $H a^{2}$ :

$$
\begin{gathered}
\phi_{H S}^{(-2)}=\phi_{H S}^{(-2)}\left(z_{H S}\right), \\
u_{H}^{(-2)}=\frac{d \phi_{H S}^{(-2)}}{d z_{H S}},
\end{gathered}
$$

Order $H a^{l}:$

$$
\phi_{H S}^{(-1)}=\phi_{H S}^{(-1)}\left(z_{H S}\right),
$$




$$
\begin{gathered}
u_{H S}^{(-1)}=\frac{d \phi_{H S}^{(-1)}}{d z_{H S}}+f^{\prime}\left(z_{H S}\right) \frac{d u_{H S}^{(-2)}}{d z_{H S}} \exp \left[y_{H S} / L^{2}\left(z_{H S}\right)\right] \\
j_{y, H S}^{(-1)}=\frac{f^{\prime 2}\left(z_{H S}\right)}{L^{2}\left(z_{H S}\right)} \frac{d u_{H S}^{(-2)}}{d z_{H S}} \exp \left[y_{H S} / L^{2}\left(z_{H S}\right)\right] \\
j_{z, H S}^{(-1)}=\frac{f^{\prime}\left(z_{H S}\right)}{L^{2}\left(z_{H S}\right)} \frac{d u_{H S}^{(-2)}}{d z_{H S}} \exp \left[y_{H S} / L^{2}\left(z_{H S}\right)\right]
\end{gathered}
$$

For proper matching with the core, it is also necessary to consider conservation of current in the layer to $O(1)$. From Eq. (4.17) follows

$$
\frac{\partial}{\partial y_{H S}}\left[j_{y, H S}^{(0)}-f^{\prime}\left(z_{H S}\right) j_{z, H S}^{(0)}\right]=-\frac{\partial_{y, H S}^{(-1)}}{\partial z_{H S}}
$$

Integrating this equation over the layer and using the matching conditions with the core gives

$$
j_{y, C}^{(0)}-f^{\prime}(z) j_{z, C}^{(0)}=\frac{d}{d z}\left[f(z) \frac{d u_{C}^{(-2)}}{d z}\right] \text { at } y=f(z) \text {. }
$$

\subsection{Further discussion of the core}

Substituting (4.12) into (4.25) and integrating the result with respect to $z$, gives the expression for the core current:

$$
j_{z, C}^{(0)}=-\frac{f^{\prime}}{f} \frac{d u_{C}^{(-2)}}{d z} .
$$

In the above the integration constant has been set to zero, since it equals the value of the total current of $O(1)$.

Now, substituting (4.27) into (4.10) gives the second-order equation for the $O\left(\mathrm{Ha}^{2}\right)$ core velocity:

$$
\frac{d^{2} u_{C}^{(-2)}}{d z^{2}}+\frac{f^{\prime}}{f} \frac{d u_{C}^{(-2)}}{d z}=-1
$$

Integrating this equation once gives

$$
\frac{d u_{C}^{(-2)}}{d z}=\frac{1}{f}\left\{a_{1}-\int_{0}^{z} f(t) d t\right\}
$$

where $a_{1}$ is an integration constant, which is proportional to friction in the core estimated at $z=0$. It is shown below that there is no parallel layer to $O\left(H a^{2}\right)$ at $z=0$, and therefore, this friction is estimated directly on the plate. Then Eqs. (4.29) and (2.29) yield 


$$
a_{1}=\frac{1}{2} A=\int_{0}^{1} f(t) d t
$$

and therefore,

$$
\frac{d u_{c}^{(-2)}}{d z}=\frac{1}{f} \int_{z}^{1} f(t) d t
$$

Finally, integrating (4.31) and using the no-slip condition gives

$$
u_{C}^{(-2)}(z)=\int_{0}^{z} \frac{d \zeta}{f(\zeta)} \int_{\zeta}^{1} f(t) d t .
$$

The remaining flow variables in the core are:

$$
\begin{gathered}
\phi_{C}^{(-2)}(z)=\int_{0}^{z} \frac{(z-\varsigma) d \varsigma}{f(\varsigma)} \int_{\zeta}^{1} f(t) d t . \\
j_{z, C}^{(0)}=-\frac{f^{1}}{f^{2}} \int_{z}^{1} f(t) d t, \\
j_{y, C}^{(0)}=y\left\{\frac{f^{\prime} f-2 f^{2}}{f^{3}} \int_{z}^{1} f(t) d t-\frac{f}{f}\right\} .
\end{gathered}
$$

It should be noted that there are no singularities in the expressions for the core variables as $z \rightarrow 1$. All of them tend to a finite limit. In particular, $j_{z, C}^{(0)}(1)=\frac{1}{3}$.

Consider now the behaviour of the core variables as $z \rightarrow 0$. One gets

$$
u_{C}^{(0)}(0)=0, \phi_{C}^{(0)}(0)=0, \quad j_{z, C}^{(0)}(0)=\frac{A \cot \theta}{2 d^{2}} .
$$

If $\theta \neq \frac{1}{2} \pi$, the $z$-component of the core current does not satisfy the second boundary condition in (2.13) at the insulating plate, and this leads to the formation of the parallel layer $P$, in which the Lorentz force is balanced by the viscous term caused by the variation of the velocity $O(H a)$. If $\theta=\frac{1}{2} \pi$, the normal component of current vanishes at $z=0$, and no parallel layer appears to $O(H a)$.

\subsection{Results}

The mechanism for the appearance of the $O\left(\mathrm{Ha}^{2}\right)$-velocity in the core is described at the beginning of Sec. 4.1. The composite asymptotic expansion for the induced magnetic field is

$$
b^{c}=\frac{f}{f^{2}} \int_{z}^{1} f(t) d t\left\{y-f \exp \left[H a \frac{y-f}{1+f^{2}}\right]\right\},
$$


and the electric current lines corresponding to (4.37) are shown in figure 7. The key in the mechanism above is again the fact that the core velocity, which does not vary along the magnetic field lines, must satisfy the zero-traction condition at the free surface. The profile of the core velocity is shown in figure 8 .

It is important to note that in contrast to the transverse field, the flow in the bulk of the cross-section is viscous. Therefore, to the leading order the "core" occupies the whole crosssection, and there are no boundary layers. 


\section{CONCLUSIONS}

Fully developed magnetohydrodynamic flow in a rivulet on an inclined plate has been considered for a partially wetted and electrically insulating plate. An asymptotic solution of the problem for high values of the Hartmann number has been obtained.

If the magnetic field is transverse to the plate, the flow in the core is determined by the balance of the Lorentz force and the gravity. The Hartmann layer is formed at the plate, which allows high electric current to circulate in the rivulet's cross-section. This leads to the reduction of the fluid velocity, the latter being proportional to $B_{0}{ }^{-1}$.

If the magnetic field is parallel to the plate, the Lorentz force alone cannot balance the gravity, and the core is viscous. The fluid velocity is independent of the magnetic field induction, and is of the same order as that in a flow without the field.

For both field directions (i) the velocity does not vary along the magnetic field lines; (ii) there is a lower-order Hartmann layer at the free surface, which is formed to relax traction at the free surface, produced by the core flow.

\section{Acknowledgement}

This work is supported by the Office of Fusion Energy Sciences, U.S. Department of Energy, under Contract No. W-31-109-ENG-38. 
1. R. Mattas, "ALPS - Advanced Limiter-Divertor Plasma-Facing Systems", Presented at $5^{\text {th }}$ Int. Symposium on Fusion Nuclear Technology, Rome, Italy, 19-24 September, 1999.

2. S. Molokov and C.B. Reed, "Review of free-surface MHD experiments and modelling," ANL Report No, ANL/TD/TM99-08.

3. M.Ye. Lebedev, B.S. Fokin and V.V. Yakovlev, "MHD flow with a free surface on the tokamak contact divertor device," Heat Transfer Research, 24, 318 (1992).

4. S. Oshima, R. Yamane, Y. Mochimaru and K. Sudo, "Investigation on film flow of a conducting fluid in a transverse magnetic field (2nd report, rivulet in a non-uniform magnetic field)," Bull. JSME., 253, 2078-2085 (1986).

5. S. Oshima and R. Yamane, "Shape-control of liquid metal free surfaces by means of a static magnetic field," In: Magnetohydrodynamics in Process Metallurgy, (J.Szekely, J.W.Ewans, K.Blazek, N.El-Kaddah, eds., The Minerals, Metals \& Materials Society), 251-259 (1991).

6. V.V. Baranov, I.A. Evtushenko, I.R. Kirillov, E.V. Firsova and V.V. Yakovlev, "Liquid metal film flow for fusion applications," Magnetohydrodynamics, 30, 460-467 (1994).

7. I. Bucenieks, O. Lielausis, E. Platacis and A. Shishko, "Experimantal study of liquid metal film and jet flows in a strong magnetic field," Magnetohydrodynamics, 30, 219-230 (1994).

8. R. Moreau, Magnetohydrodynamics (Kluwer, Amsterdam, 1990).

9. R.F. Allen and C.M. Biggin, "Longitudinal flow of a lenticular liquid filament down an inclined plate," Phys. Fluids 17, 287-291 (1974).

10. G.D. Towell and L.B. Rothfield, "Hydrodynamics of rivulet flow," J. Am. Inst. Chem. Eng. 12, 972-980 (1966).

11. T. Alboussiere, J. P. Garandet and R. Moreau, "Buoyancy-driven convection with a uniform magnetic field. Part 1. Asymptotic analysis," J. Fluid Mech. 253, 545-563 (1993). 
12. S. Aleksandrova and S. Molokov, "Three-dimensional buoyant convection in a rectangular cavity with differentially heated walls in a strong magnetic field," In: EPM2000, Nagoya, Japan (submitted) (1999).

13. M.D. Cowley, "Parallel wall layers in buoyancy-driven MHD flows," unpublished manuscript (1999).

14. J. C. R. Hunt and G. S. S. Ludford, "Three-dimensional MHD duct flows with strong transverse magnetic fields. Part 1. Obstacles in a constant area channel," J. Fluid Mech. 33, 693-714 (1968). 


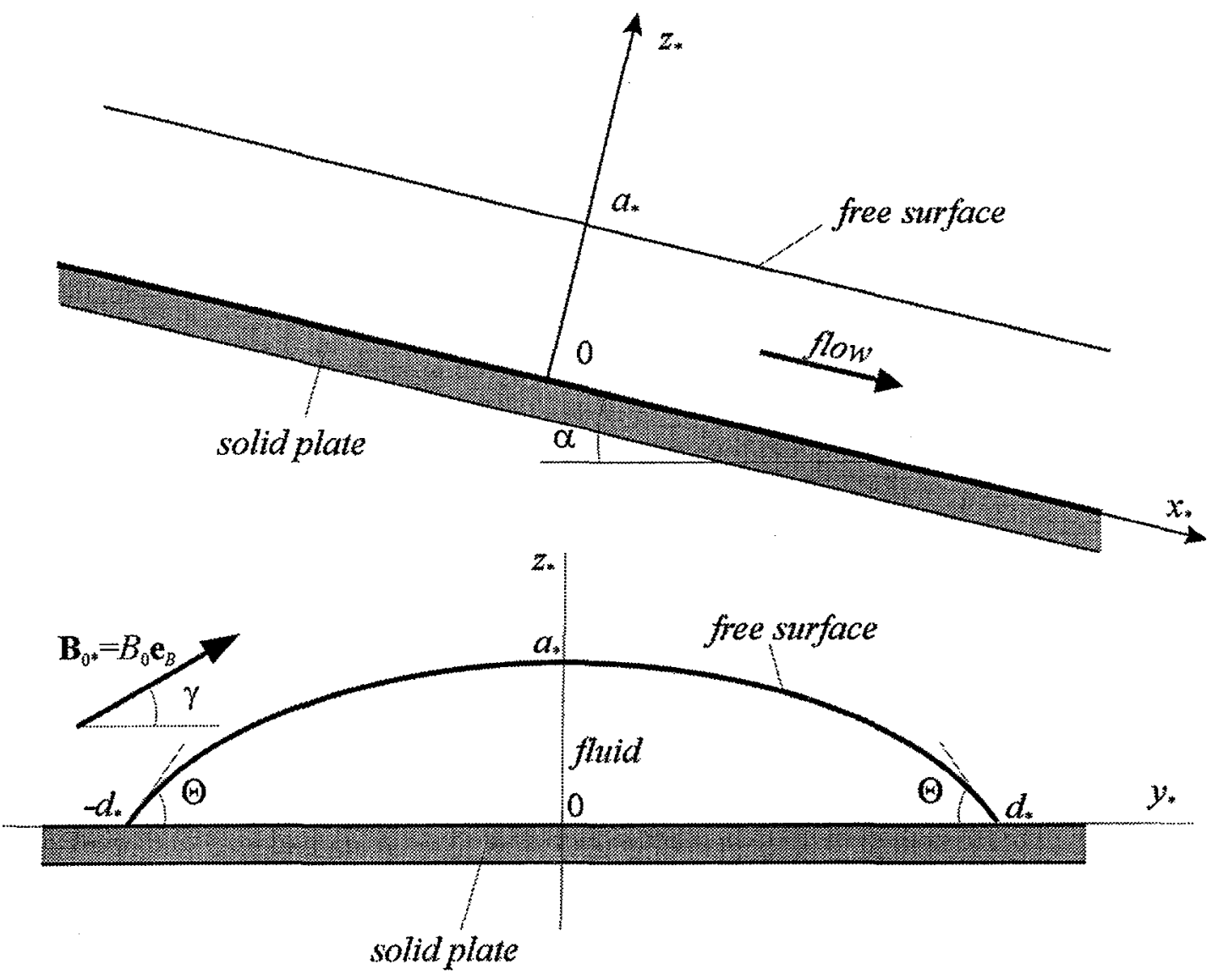

Figure 1. Schematic diagram of the flow in a rivulet.

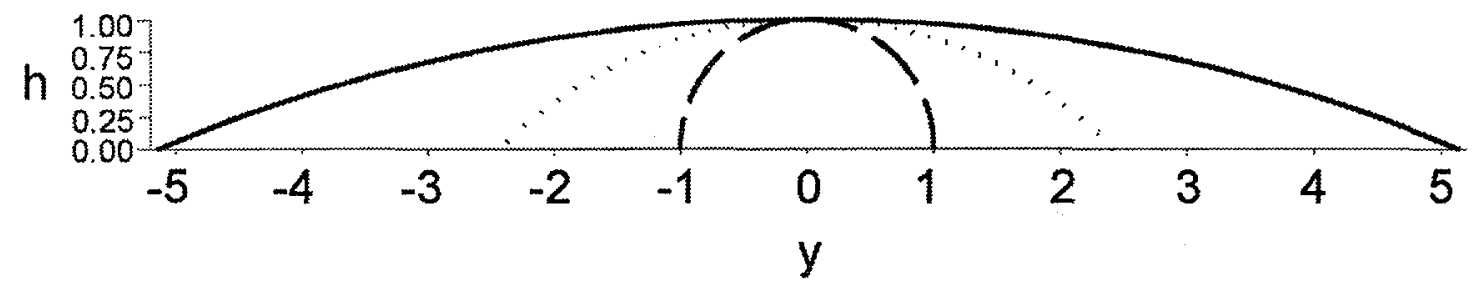

Figure 2. Rivulet shape for $k=0.01$ and for three values of the contact angle, $\theta: \pi / 8$ (solid line), $\pi / 4$ (dotted line), $\pi / 2$ (broken line). 


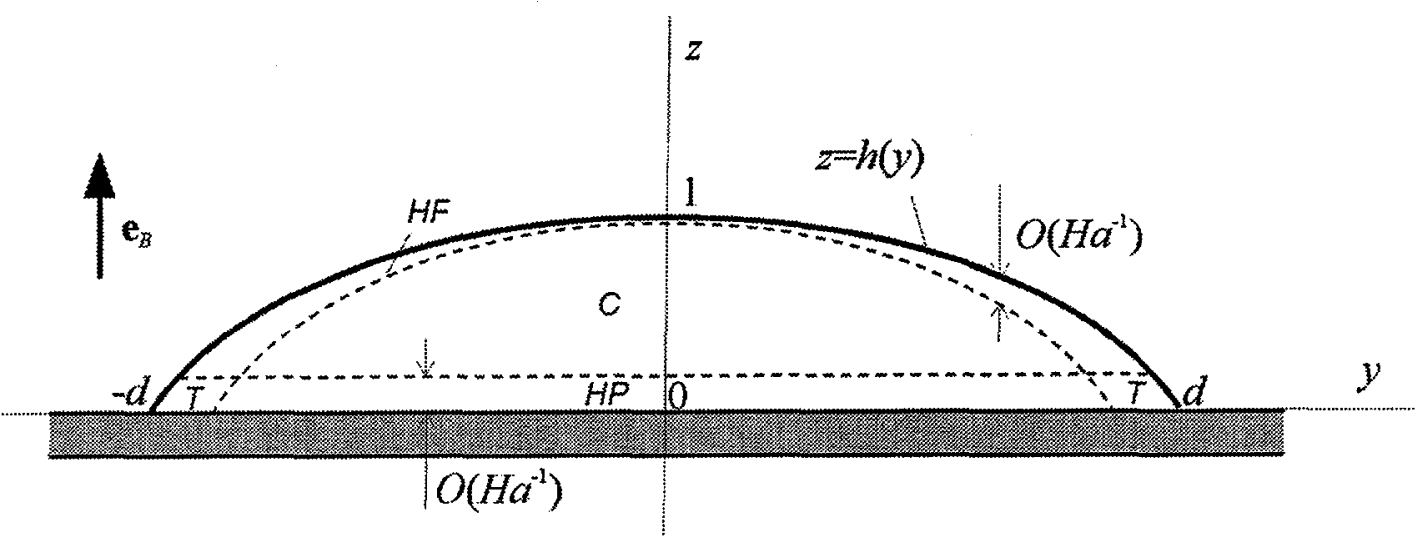

Figure 3. Flow in a transverse field: flow subregions at high Hartmann number.

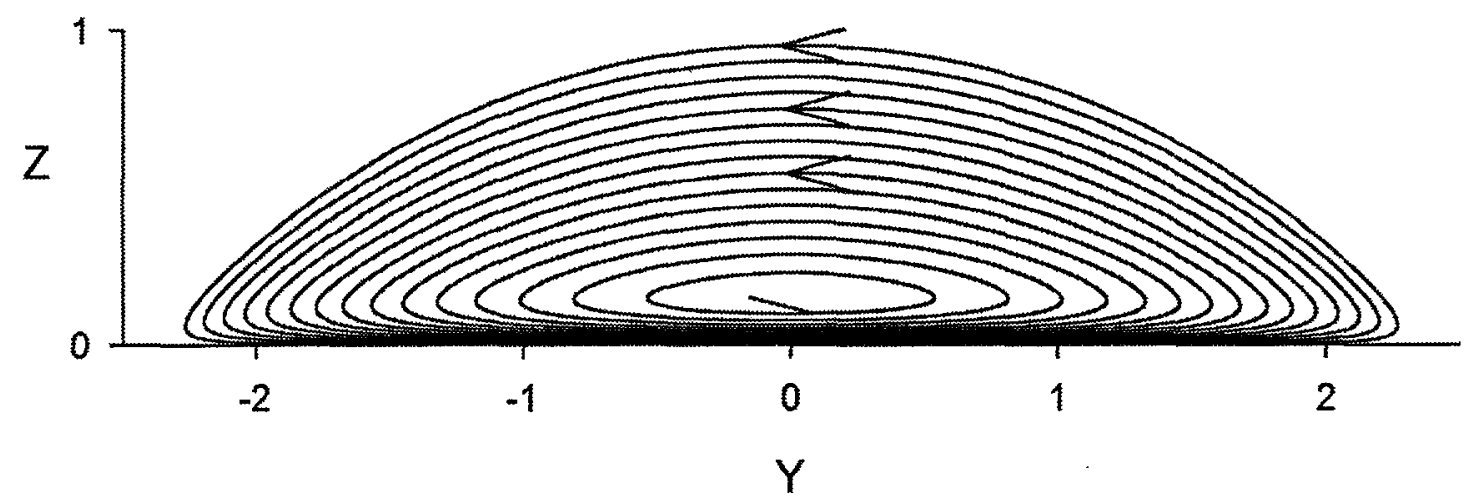

Figure 4. Flow in a transverse field: electric current lines for $k=0.01, \mathrm{Ha}=20$, and $\theta=\pi / 4$. 


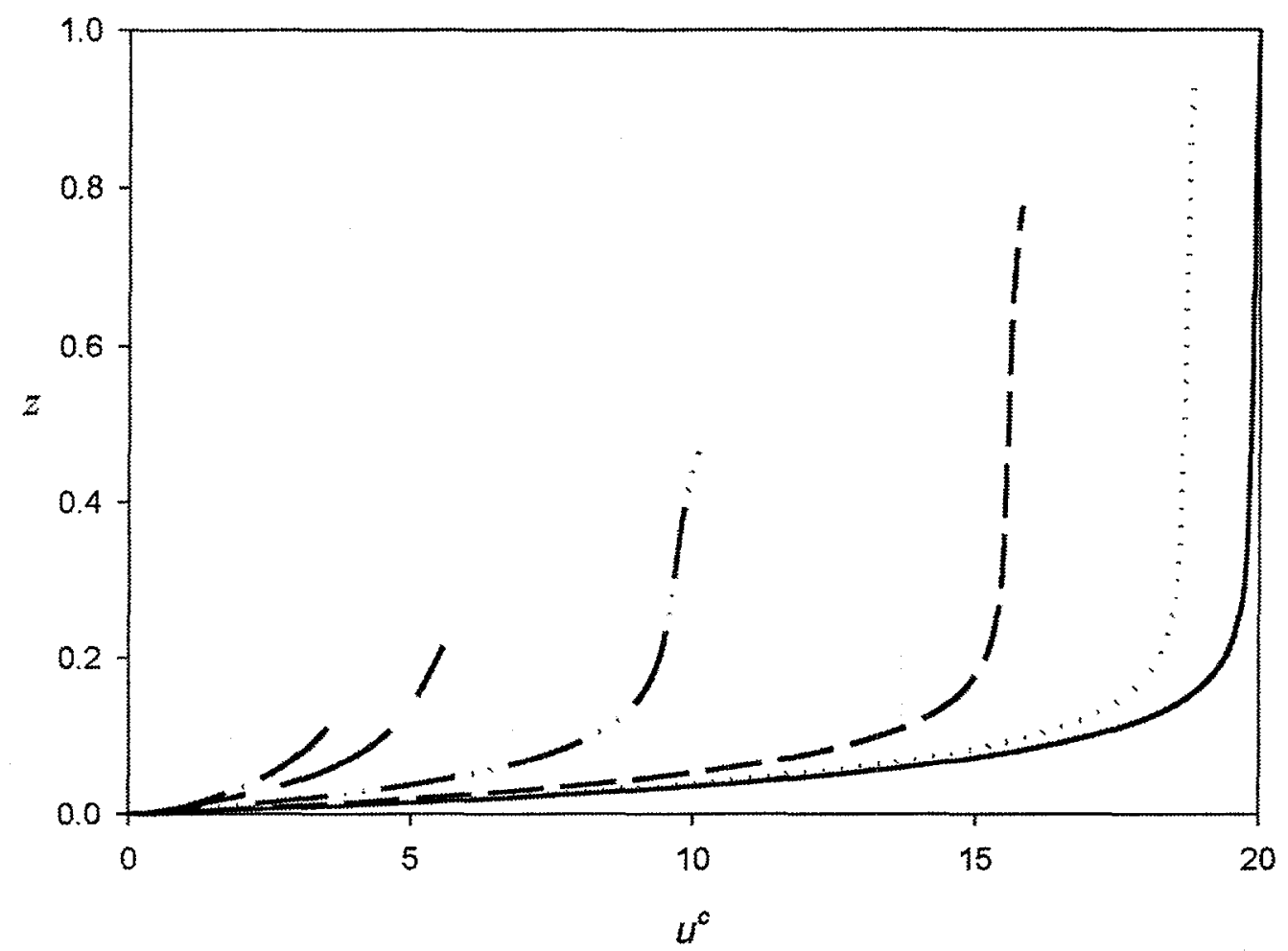

Figure 5. Flow in a transverse field: velocity profiles for $\mathrm{k}=0.01, \mathrm{Ha}=20$, and $\theta=\pi / 4$ at $y=0$ (solid line), $y=0.65$ (dotted lines), $y=1.22$ (broken line), $y=1.85$ (dashed-double-dotted line), $y=2.19$ (long dashed line), 2.32 (dashed-dotted line).

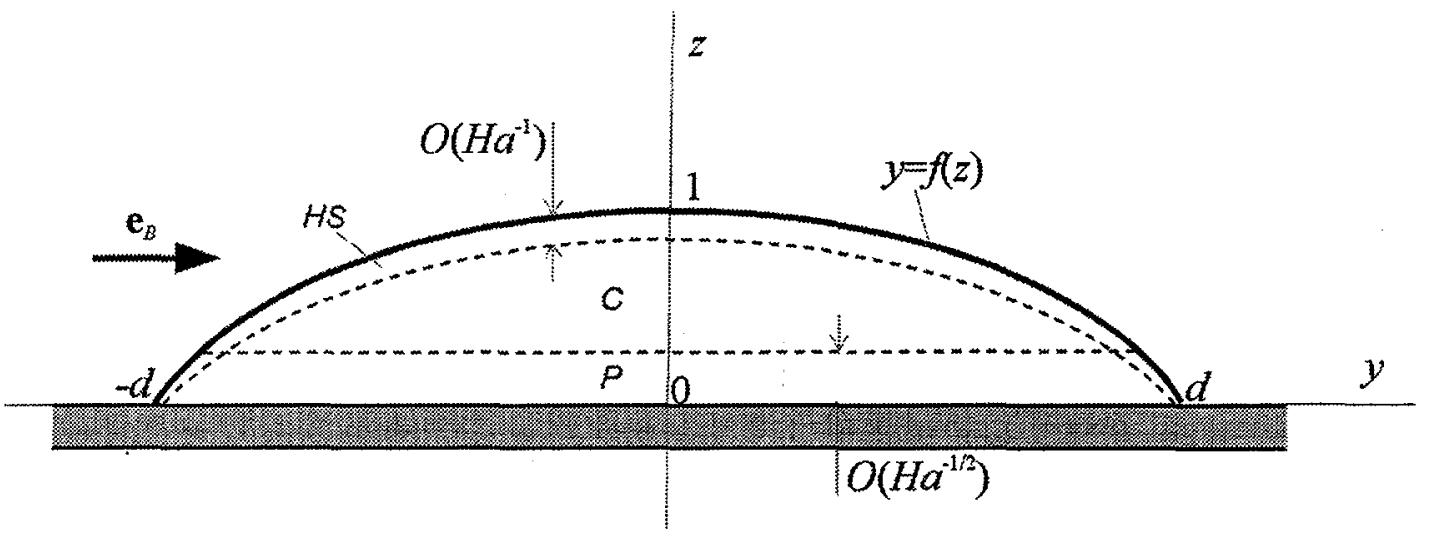

Figure 6. Flow in a parallel field: flow subregions at high Hartmann number. 


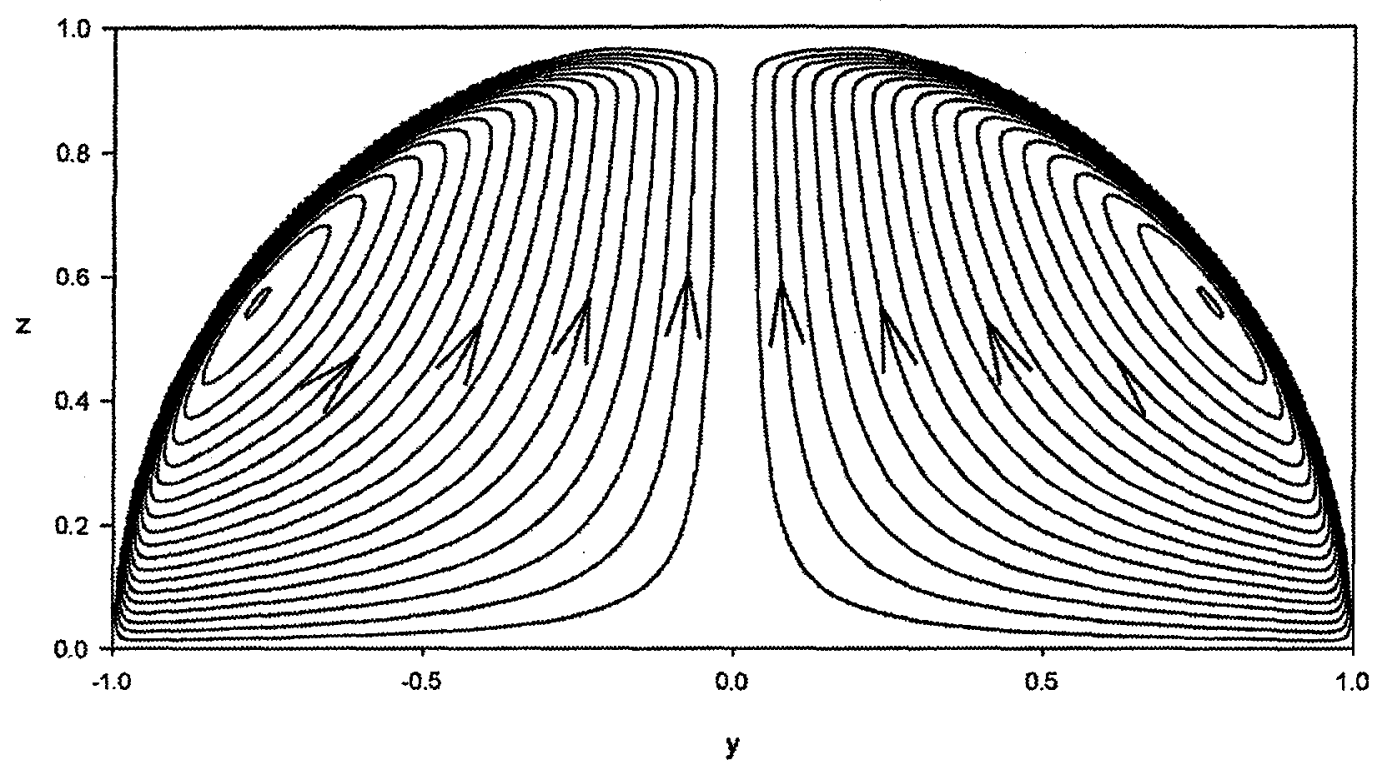

Figure 7. Flow in a parallel field: electric current lines for $k=0.01, \mathrm{Ha}=100$, and $\theta=\pi / 2$.

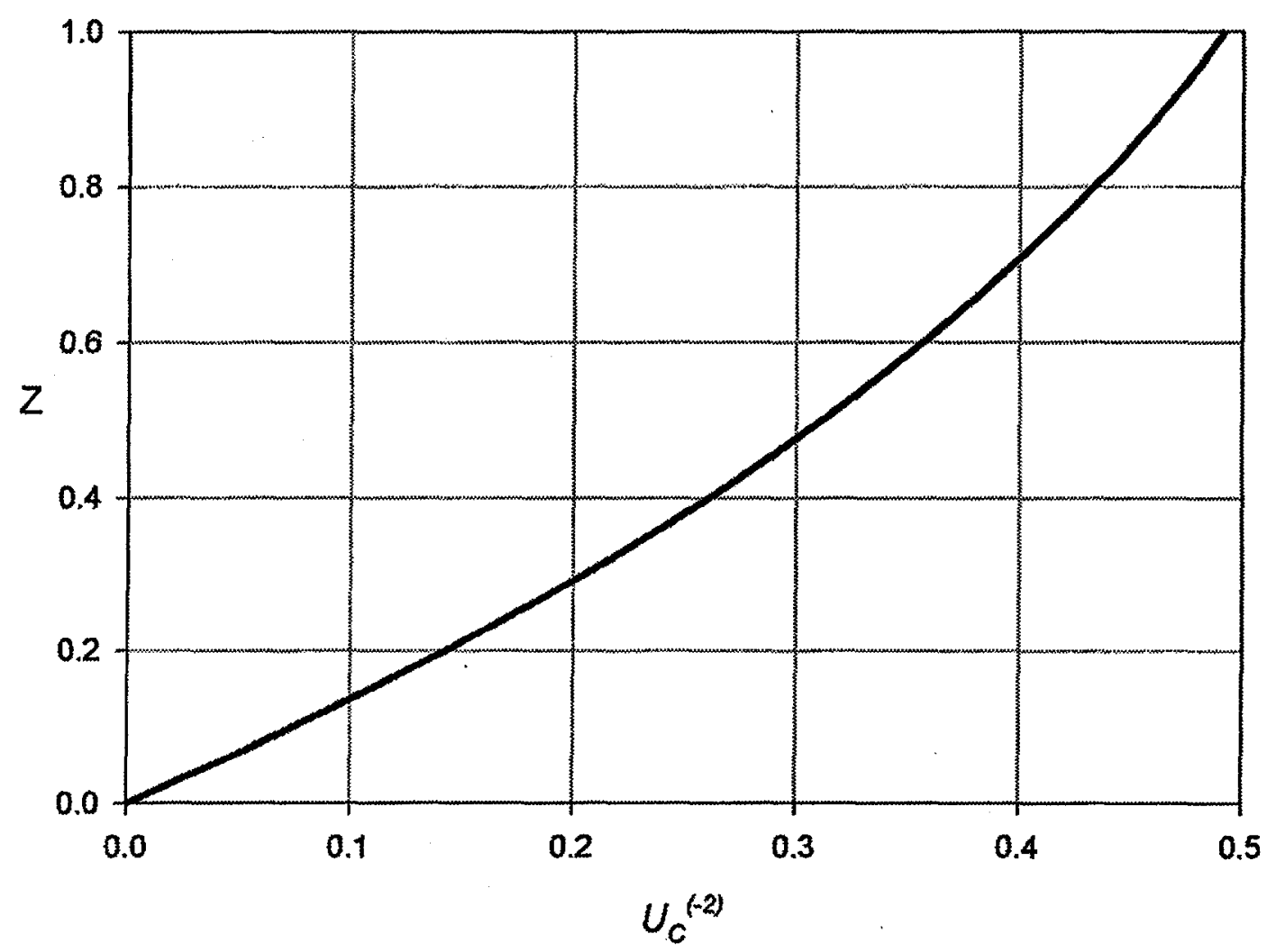

Figure 8. Flow in a parallel field: core velocity profile for $\mathrm{k}=0.01, \mathrm{Ha}=100$, and $\theta=\pi / 2$. 


\section{DISTRIBUTION LIST FOR ANL/TD/TM00-12}

\section{Internal}

S. Bhattacharyya

R. Mattas

A. Hassanein

J. Brooks

T. Hua

D.L. Smith

D.-K. Sze

K. Natesan

C. Reed (5)

FPP Files (10)

TIS Files

\section{External}

DOE/OSTI, for distribution (2)

ANL-E Library

ANL-W Library

C. Baker, University of California, San Diego, CA

S. Berk, U.S. Department of Energy, Germantown, MD

S.A. Cohen, Princeton Plasma Physics Laboratory, Princeton, NJ

R. Conn, University of California, San Diego, CA

O. Filatov, Efremov Scientific Research Institute, St. Petersburg, Russia

R. Goldston, Princeton Plasma Physics Laboratory, Princeton, NJ

I. Kirillov, Efremov Scientific Research Institute, St. Petersburg, Russia

S. Malang, Forschungszentrum Karlsruhe, Karlsruhe, Germany

K. McCarthy, Idaho National Engineering and Environmental Laboratory, Idaho Falls, ID

S. Molokov, Coventry University, Coventry, United Kingdom

F. Najmabadi, University of California, San Diego, CA

R. Nygren, Sandia National Laboratories, Albuquerque, NM

J. Reimann, Forschungszentrum Karlsruhe, Karlsruhe, Germany

M. Tillack, University of California, San Diego, CA

J. Vetter, Forschungszentrum Karlsruhe, Germany

F.W. Wiffen, U.S. Department of Energy, Germantown, MD

Bibliothek, Max-Planck-Institute fur Plasmaphysik, Germany

C.E.A. Library, Fontenay-aux-Roses, France

Librarian, Culham Laboratory, England

Thermonuclear Library, Japan Atomic Energy Research institute, Japan

University of Illinois, Fusion Studies Laboratory

University of Illinois, Grainger Engineering Library Information Center 